\title{
Carnets
}

Revue électronique d'études françaises de l'APEF

Deuxième série - 7| 2016

Plurilinguisme et migrations dans la littérature de langue française

\section{Plurilinguisme et migrations dans Nord Perdu de Nancy Huston}

\section{Britta Benert}

\section{(2) OpenEdition}

Journals

Édition électronique

URL : http://journals.openedition.org/carnets/918

DOI : 10.4000/carnets.918

ISSN : 1646-7698

Éditeur

APEF

Référence électronique

Britta Benert, «Plurilinguisme et migrations dans Nord Perdu de Nancy Huston », Carnets [En ligne], Deuxième série - 7 | 2016, mis en ligne le 31 mai 2016, consulté le 19 avril 2019. URL : http://

journals.openedition.org/carnets/918; DOI : 10.4000/carnets.918

Ce document a été généré automatiquement le 19 avril 2019

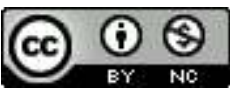

Carnets est mis à disposition selon les termes de la licence Creative Commons - Atribution - Pas d'utilisation commerciale 4.0 International. 


\title{
Plurilinguisme et migrations dans Nord Perdu de Nancy Huston
}

\author{
Britta Benert
}

L'intérêt inlassablement croissant que voue la critique au phénomène du plurilinguisme littéraire est récent dans l'histoire littéraire et sans doute à mettre en rapport avec les bouleversements politiques et culturels engendrés par la chute du mur de Berlin il y a 25 ans. Dans ce contexte de mondialisation, les écrivains « migrants» ou auteurs « venus d'ailleurs » (Delbart, 2005; Mathis-Moser et Mertz Baumgartner, 2012) jouissent d'une nouvelle curiosité non exempte d'ambiguité, par exemple, lorsque la catégorisation en tant qu'« autre » tend à stigmatiser l'auteur et son œuvre pour finir par empêcher leur reconnaissance littéraire à part entière. Nancy Huston ayant été récompensée par les plus prestigieux prix littéraires existant en France, connaît quant à elle une consécration certaine, un succès qui se reflète dans de fines lectures auxquelles a donné lieu son œuvre littéraire. Dans ce cadre, qu'en est-il du texte Nord perdu, paru en 1999? Largement sollicité par la critique, l'essai est parfois cité de façon décontextualisée, avec comme conséquence, peut-être, de muer Nord perdu en un texte aux prises de positions nettes et immuables. En d'autres termes, en cas de références parcellaires, le risque existe que celles-ci ne permettent de saisir la foncière ambiguïté de l'essai hustonien, fondée sur la présence simultanée d'une multitude de voix cacophoniques énoncées par un unique je. Je proposerai ainsi dans un premier temps de lire Nord perdu comme texte littéraire (et non documentaire), en dégageant ses procédés et ficelles et en appuyant ma lecture sur le concept d'une " poétique de la migrance » (Mathis-Moder et Mertz Baumgartner, 2012). Je m'interrogerai dans un deuxième temps sur la manière paradoxale dont Nord perdu traite de la question de «l'inappropriable linguistique » (Jenny, 2005), consolidant, là encore, l'inscription du texte hustonien dans une « poétique de la migrance ». Enfin, en guise de conclusion, je présenterai une brève réflexion sur les limites de la classification en général, ainsi que, plus particulièrement, sur le défi que peut constituer le travail consacré aux auteurs classés « venus d'ailleurs » avec des étudiants. 


\section{Nord perdu comme " poétique de la migrance »}

2 une perspective générale, de définir « une poétique de la migrance ». Elles écrivent :

(...) les écrits de nombreux auteurs migrants témoignent d'une hybridité textuelle et générique : au lieu de créer des textes homogènes, unifiés et linéaires, ils jouent avec la fragmentation, la contradiction, l'hétérogénéité et la pluralité des voix et des perspectives. Le mélange des genres est fréquent, les fragments d'autobiographie, de critique, de textes journalistiques, d'entrevues ou d'émissions de télé s'intercalant et rompant le rythme du récit. Intertextualité et intermédialité sont à l'ordre du jour et s'inscrivent majoritairement dans une logique de la différence et de la transculture (Mathis-Moser et Mertz Baumgartner, 2012 : 15).

Cette définition peut se lire, me semble-t-il, comme une synthèse efficace de la poétique à l'œuvre dans Nord perdu dont les thématiques prépondérantes sont la migration et les questions identitaires qui en sont le corollaire, présentées par le biais d'une protagoniste «migrante » et le double regard qu'elle porte sur son pays d'origine d'une part, sur le pays d'accueil de l'autre. Aussi, dans ce qui suit, partirai-je de trois critères clés que sont l'hybridité textuelle, le mélange des genres et l'intertextualité afin de penser ces éléments à l'égard du texte hustonien.

\section{L'hybridité générique}

Nord perdu enchâsse de nombreux passages à caractère autobiographique, de longues citations d'auteurs, de commentaires de journaux, de réflexions linguistiques, sociolinguistiques, poétologiques et d'allusions à des émissions radiophoniques («Le masque... » « ... et la Plume » forment les intitulés de deux chapitres consécutifs de Nord Perdu) de sorte que l'hybridité générique apparaisse comme l'un des traits dominants du texte. Il en résulte un impossible classement qui participe au caractère foncièrement fragmenté de Nord perdu et contribue parallèlement au fait que le lecteur est amené à ne cesser de perdre le fil - Huston orchestrant ici une mise en abyme entre le désarroi du «je» du texte et celui de son lecteur, non sans rappeler Nabokov (ou d'autres auteurs plus près de nous, ...) et le goût de ce dernier pour les fausses pistes.

\section{L'hybridite textuelle}

5 Les premières pages $d u$ texte hustonien sont particulièrement représentatives de l'hybridité textuelle, rendue par un jeu omniprésent avec la fragmentation et avec la pluralité des voix et des perspectives. L'observation de l'ouverture permet par ailleurs de souligner le subtil agencement littéraire du texte. Regardons: Nord perdu ouvre sur une dédicace («Pour Lorne, mon frère de sang, d'âme et de langues»); celle-ci est accompagnée d'une première citation (de T.S. Eliot : « Home is where you start from »); à la page suivante, le titre de l'ouvrage est répété et orné de deux nouvelles épigraphes versifiées ; la page intitulée « Envoi » ressemble à un premier chapitre, une ouverture au texte donc, avec un marquage typographique de la première lettre qui, en confortant la présence d'une organisation en chapitres, indiquerait le début d'un premier. "Envoi » serait alors à comprendre sportivement, dans le sens de "coup d'envoi » pour annoncer l'une des strates présente surtout dans une deuxième partie, une strate qui inscrit le texte 
dans une actualité (politique et culturelle), avec des références à la Coupe du monde de football et à la victoire des Bleus en été 1998 ("La Mosäique arrogante », Huston, 1999 : 82) ainsi que la mention de personnalités et ou d'événements du monde politique et culturel de la France (les chapitres "La Détresse de l'étranger ", «Relativement relatif » citent Jean-Marie Le Pen, Jean Toubon, Jean Tiberi, le scandale Elf, Michel Houellebecq, Gérard Depardieu, les Guignols, ...). Si la table en fin de texte ne semble laisser la place à aucun doute - il s'agirait bien d'un écrit composé de 14 chapitres/unités textuelles -, l'ouverture sur une nouvelle citation ( $J E$ NE ME PLAIS PAS. Oui. », 1999 : 11) instaure une continuité avec le péritexte (dédicace et épigraphes) ayant pour effet, d'une part, de brouiller la délimitation entre texte et péritexte, d'autre part, de rappeler la polysémie du terme envoi : aussi ne fait-il aucun doute que Huston rend hommage à Sviatoslav Richter et à Romain Gary ; certes, sans recours à la versification - pas de quatrain final ici -, mais en plaçant sa double dédicace à l'ouverture de son texte, sous forme de neuf lignes en prose.

6 Je voudrais m'arrêter sur cet incipit (« 'JE NE ME PLAIS PAS. Oui'. C'est Sviatoslav Richter qui parle»), en dégager quelques ruptures et fragmentations, comme étant autant de reflets des ruptures et fragmentations de l'ensemble du texte :

- En s'inscrivant dans une continuité avec les épigraphes des deux pages qui précèdent, le « Je » pourrait se prêter à être confondu avec l'auteur de l'essai (Nancy Huston et/ou la voix narrative), mais ne doit pas l'être ... à moins que la potentielle hésitation soit une invitation à le faire? ;

- «JE NE ME PLAIS PAS. Oui » est également désorientant par la juxtaposition d'une proposition négative où, grammaticalement, on s'attendrait plutôt à un renforcement par une nouvelle négative (qui donnerait "Je ne me plais pas. Non»). Le «Oui» (effectif, affirmatif...) introduit un écart par rapport à cette attente dictée par une norme grammaticale (française) et a pour conséquence d'ébranler la négativité de l'affirmation (!) négative ;

- Si l'on prend en considération qu'en langue russe (et contrairement donc au français), la négation peut/doit être renforcée par une affirmative, c'est un autre écart qui se dégage, se situant, celui-ci, entre le français et le russe. La proposition "Je ne me plais pas. Oui.» reposerait ainsi sur une traduction littérale qui fait que le russe transparaît à travers la proposition française assurant d'emblée la polyphonie au texte.

- Le vertige peut également s'installer à l'égard de la porosité entre l'écrit et oral : ce serait Sviatoslav Richter qui parle («C'est Sviatoslav Richter qui parle» peut-on lire); or, avec notre texte, c'est une narratrice qui prête sa voix au pianiste en le citant à l'écrit. En réalité c'est donc elle qui "parle ", en transcrivant ses mots à lui (et en les traduisant du russe, peut-être) ; ces mots dont on ne sait pas d'ailleurs si le pianiste les a réellement prononcés ou écrits - un jour. Pour dire que l'affirmation (péremptoire) « C'est Sviatoslav Richter qui parle » peut être mise en question, doit être mise en question : a-t-il vraiment parlé ainsi ? Dans tous les cas de figures, ce n'est pas lui qui parle ici, mais un je qui écrit...

\section{L'intertextualité}

7 À considérer les seules trois premières pages de l'essai, le nombre de références est déjà remarquable. Cette presque surenchère d'envois intertextuels contribue largement elle aussi à l'hétérogénéité et au caractère abyssal du texte. Aussi est-il clair que le nom du grand Richter rappelle le rôle très important que joue la musique dans la vie de Nancy Huston en général, le piano en particulier, instrument qu'elle abandonne à son arrivée en France pour le remplacer par le clavecin. Ce changement d'instrument se superpose avec 
le changement de langue de l'auteur, qui abandonne l'anglais pour le français (1999: 64-65). Voilà d'entrée de texte un abîme de connexions qui s'offre à nous, nous invitant à creuser les possibles interrelations, jeu d'intertextualités qui se confirme par la suite devenant une marque importante du texte - c'est en effet une avalanche de noms d'auteurs qui se trouve cités dans Nord perdu, à qui Nancy Huston a consacré des écrits/ essais (Romain Gary, Samuel Beckett, Jean-Paul Sartre...).

Quant à ce jeu d'interrelations fondé sur les références intertextuelles, notons encore que l'écriture de Nord perdu coïncide avec la mort de l'éminent pianiste, décédé à Moscou en été 1997. La mort est donc présente en filigrane d'abord, puis de façon explicite par l'évocation du suicide de Romain Gary. Comme tout livre qui se respecte, l'ouverture (dédicace, épigraphes et incipit) condense bel et bien les thématiques essentielles et les procédés littéraires du texte: 1) l'interrogation identitaire du Moi (dissocié) moderne ( «Prendre plus en pitié mon propre cœur; avec/Bonté, dorénavant, contempler mon triste moi, /Avec charité; et que cet esprit tourmenté ne vive plus/Avec cet esprit tourmenté le tourmentant encore »; "Je ne me plais pas »; "Au départ, la haine de soi ») ; 2) le XXe siècle et son histoire violente qu'incarnent les deux noms de Richter et de Gary. S'y ajoute que, par le contexte historique esquissé, l'intitulé « Envoi » n'est pas sans suggérer le mot convoi : convois assassins des Nazis qui menaient à Auschwitz, le camp d'extermination étant cité dans un dernier chapitre à travers une référence à l'ouvrage Si c'est un homme de Primo Levi (« Hier ist kein Warum », Huston, 1999 : 108 ; 3) la question de l'exil, de la migration et du changement de langue, enfin, sont des expériences de ce qui fonde la condition humaine, considérées non pas comme exceptionnelles mais étant plus visibles, plus palpables, car : «En fait nous sommes tous multiples (...) l'expatrié découvre de façon consciente (et parfois douloureuse) un certain nombre de réalités qui façonnent, le plus souvent à notre insu, la condition humaine » (Huston, 1999 : 18).

Hybridité (générique et textuelle) et intertextualité forment donc des éléments structurels effectivement à l'œuvre dans Nord Perdu et permettent de saisir le texte comme étant représentatif d'une poétique de la migrance (et l'on pourrait déjà indiquer ici, fort de la mise au point que nous venons de citer, que le terme migrance est à prendre dans un sens large, voire figuré : nul besoin que le déplacement et/ou le voyage soient effectifs pour expérimenter l'éclatement de la conscience moderne ...).

\section{Moi multiple et « l'inappropriable linguistique »}

"(Oui je sais: je me dis et puis je me contredis. Je m'en fous et puis je m'en contrefous. Je cherche mes repères...alors c'est normal, non, de tourner en rond par moments ?)» (Huston, 1999 : 86). Souvenons-nous que la contradiction, évoquée dans ce passage de façon explicite, forme un autre critère de la "poétique de la migrance ». Ces propos contradictoires sont très présents à travers l'une des interrogations clés du texte, celle qui porte sur la langue ${ }^{1}$.

11 C'est en donnant voix aux différents types de rapports que l'on peut avoir à la langue que la polyphonie contradictoire orchestrée dans Nord Perdu me semble particulièrement nette. Je suivrai ici l'admirable argumentation de Laurent Jenny (2005). Sa réflexion sur la manière dont les hommes se positionnent à l'égard de la langue/des langues l'amène à identifier trois "gestes de réappropriation", celui du puriste, celui de l'apologiste du métissage et celui du forgeur du style. Ce sont là trois gestes engendrés par «l'inappropriable linguistique », c'est-à-dire formés face à l'expérience que la langue - et 
même si je la dis « mienne » - irréductiblement, m'échappe ; toute langue m'est en dernier lieu étrangère. Je montrerai dans ce qui suit que Nord perdu réfléchit sur deux de ces trois stratégies, le geste du puriste et celui du forgeur du style.

L'attitude puriste, selon Laurent Jenny et toujours en référence à Jacques Derrida, «a toujours une portée politique » (2005:4), ce qui permet de saisir la forte présence de l'actualité politique (du discours parfois militant), présent dans Nord Perdu, tel un rappel de la foncière imbrication entre langue et politique : «Les xénophobes », écrit en ce sens Nancy Huston, « avec leur message d'identification, simplistes mais ô combien rassurante, cherchent au contraire à diluer les nuances» (Huston, 1999: 37-38). On retrouve la dimension affective qui émane de la citation hustonienne dans la description, courte et efficace, que Laurent Jenny donne de l'attitude puriste. Lisons :

[la stratégie qu'emprunte le puriste] affirme la propriété de la langue, sa transparence et sa naturalité. Elle prétend à la maîtrise d'un savoir sur la langue et de la langue. Ce savoir n'est d'ailleurs pas véritablement nommable : il relève d'une intuition innée, d'une participation nationale, raciale ou mystique au 'génie' de la langue. En ce sens, le puriste se confirme dans une sorte de tourniquet logique: l'innéité de son intuition garantit la pureté de sa langue, et réciproquement la pureté de son expression conforte son enracinement identitaire. Mais cette position demeure infiniment fragile. Elle s'expose à tous les démentis et toutes les difficultés que peut lui infliger l'historicité de la langue. Le puriste ne saurait maintenir ses certitudes qu'au prix d'une négation de l'Histoire (Jenny, $2005: 4$ ).

C'est le chapitre Le Masque (intitulé polysémique - et polyphonique - puisque référant à la fois à une notion poétologique, à une image commune pour la dissimulation et au monde culturel français), qui est plus particulièrement consacré à la langue. L'idée de naturalité et d'innéité y est évoquée par une mise en opposition entre l'apprentissage «naturel » et sans accent du bébé, et « l'artifice » de l'étranger, « condamné à l'imitation consciente » (Huston, 1999: 32) et à « s'installer à tout jamais dans l'imitation, le faire-semblant, le théâtre " (Huston, 1999: 30), à mentir et à trahir donc. Trahison et mensonge renvoient ici à la perception du puriste, celui-ci est représenté d'abord par "Les Français", dont la «maîtrise » prétendue de "leur» langue (considérée au singulier!), les autorise à corriger - à condamner - tout écart à la norme grammaticale :

L'étranger, donc, imite. Il s'applique, s'améliore, apprend à maitriser de mieux en mieux la langue d'adoption... Subsiste quand même, presque toujours, en dépit de ses efforts acharnés, un rien. Une petite trace d'accent. Un soupçon, c'est le cas de le dire. Ou alors...une mélodie, un phrasé atypique...une erreur de genre, une imperceptible maladresse dans l'accord des verbes...Et cela suffit. Les Français guettent...ils sont tatillons, chatouilleux, terriblement sensibles à l'endroit de leur langue...c'est comme si le masque glissait...et vous voilà dénoncé! (Huston, 1999 : 33).

L'attitude puriste n'est pas réservée aux seuls Français. Aussi, plus loin dans le texte, est-il question d'Anglophones, Huston prêtant la conception de naturalité et de maîtrise innée de la langue à sa famille « d'origine ». Tout en reprenant l'approche normative attribuée aux Français à l'entrée du chapitre, ce passage consacré à l'expérience du retour au "pays» insiste tout particulièrement sur une vision essentialisée de la langue maternelle :

C'est ça, ta langue maternelle? T'as vu l'état dans lequel elle est? Mais enfin, c'est pas possible! Tu as un accent! Tu n'arrêtes pas d'introduire dans ton anglais des mots français. C'est ridicule! Tu fais semblant ou quoi ? Tu essaies du nous épater avec ta prestigieuse parisianité ? Allez, ça ne marche pas, on n'est pas dupes, on sait que tu es anglosaxophone comme tout le monde...Parle normalement! Arrête de 
faire des fautes! Arrête de chercher tes mots! Tu les as, tes mots, tu les as avalés avec le lait maternel, comment oses-tu faire mine de les avoir oubliés? Parle tout droit, enfin, parle naturel, parle anglais !!!! (Huston, 1999 : 39-40). canadienne ou américaine, ...), mais au fond une stratégie que l'on décide d'employer, que Nancy Huston invente le concept des «monolingues impatriés » (Huston, 1999: 37) désignant ainsi tout ceux n'ayant pas fait l'expérience de l'étrangeté inhérente à toute langue. Dans cette perspective, l'insistance, dans ce chapitre, sur l'accent est symptomatique. C'est en effet de façon récurrente que l'emploi se fait, la notion figurant à chaque page. Si l'accent incarne l'idée d'écart, le « puriste » ne peut que se ranger du côté des lectures traditionnelles du phénomène, comme étant un écart «condamnable » à l'égard d'une norme linguistique, d'une vision essentialisée de la langue qui ne peut exister qu'au singulier. Va de pair avec la perception de la langue comme réalité homogène et unique la fixation nationale, également mise en avant par Laurent Jenny dans le sens où l'identité, selon l'attitude puriste, se réduit à l'appartenance nationale :

(...) dès que vous la leur fournissez [aux Français], cette information se cristallisera dans leur esprit, se figera, deviendra votre trait le plus saillant, la qualité qui, entre toutes, vous définit et vous décrit. Vous serez la Russe, le Néo-Zélandais, le Sénégalais, la Cambodgienne et ainsi de suite (un magazine respectable a récemment qualifié la cinéaste Agnieska Hollande de 'Polonaise de service'; un autre a cru élégant de commencer une critique d'un de mes livres par la phrase : 'Elle est morose, notre Canadienne') ... alors que, bien sûr, chez vous, votre nationalité était l'air même que vous respiriez, autant dire qu'elle n'était rien (Huston, 1999 : 34).

geste du forgeur du style fait partie de l'acceptation poétologique du Masque; il est plus particulièrement pensé dans le chapitre intitulé «La Plume». À l'inverse du "puriste », le forgeur du style défend l'idée d'une pluralité inhérente à la langue, selon la formule du linguiste Mario Wandruszka pour qui « chaque langue est plusieurs langues » et bien sûr selon les conceptions poétologiques de Marcel Proust. Ce dernier incarne le geste du forgeur du style et constitue une référence clé de Huston, ce dont probablement l'intitulé de l'essai témoigne déjà (Nord perdu/temps perdu) ${ }^{2}$. Les affirmations «(...) est important ce qui est traduisible » (Huston, 1999: 90, en italiques dans le texte) et «Il vous reste toujours l'écriture " qui clôt le chapitre (Huston, 1999: 92) sont des prises de position en faveur d'un rapport esthétique à la langue. Ce rapport esthétique implique une dimension éthique : «Au fond, me semble-t-il, l'étrangéité est une métaphore du respect que l'on doit à l'autre » (Huston, 1999 : 37). Dans un même ordre d'idées éthique, citons encore la fin du chapitre «Le Masque » et sa question (rhétorique) finale : «Serait-ce que ... y compris dans ma propre langue ... je ne me supporte qu'étrangère, dotée d'un accent? " (Huston, 1999 : 41-42). Cela revient à référer à « l'inappropriable linguistique ", au caractère irréductiblement étranger de toute langue qui toujours échappe et à saisir cette altérité comme moteur de toute écriture.

Quant aux trois stratégies identifiées (puriste, apologiste du métissage, le forgeur du style), par Laurent Jenny, il est précisé: "On pourrait tracer une opposition fondamentale entre ceux qui dénient l'impropriété et ceux qui s'efforcent de lui accorder un statut idéologique ou esthétique » (Jenny, 2005 : 4). Puriste d'un côté donc, forgeur du style et apologiste du métissage de l'autre, dans Nord Perdu, c'est sans crier gare que la voix narratrice mêle au sein d'un même passage l'une et l'autre attitudes pour ne cesser de brouiller toute idée de frontière absolue, cassant les dichotomies qui s'avèrent, tout 
compte fait, des béquilles de la pensée bien trop commodes ${ }^{3}$. C'est dans ce jeu avec la fragilité des oppositions que se situe la foncière ambiguïté du texte hustonien et qu'émane son écriture paradoxale.

\section{En guise de conclusion : Penser/Classer}

18 À Strasbourg, à ma demande sur si le dernier roman de notre auteur est bien disponible, le libraire me renvoie au rayon de littérature française, puisque, précise-t-il, «Nancy Huston a aussi écrit en français ", sous-entendant par là qu'elle aurait pu se trouver rangée du côté du "monde anglophone", autre espace distingué par la librairie. En traversant le Rhin, chez le libraire de la petite ville allemande de Kehl, c'est un autre habitus que je rencontre. Ici, pas de distinction «nationale » ou par « aires linguistiques », mais une stricte organisation alphabétique, Nancy Huston entre Ödön von Horváth et Siri Hustvedt. Il est clair que Nancy Huston fait partie de «ces êtres de frontières, ces inclassables, ces cosmopolites (...) » décrits par Julia Kristeva (1995 : 43), tout comme il ne fait pas de doute que l'une et l'autre tentative de classification impliquent des limitations discutables: aussi, du côté du libraire allemand, peut-on voire guetter un danger d'« annexion » par la littérature allemande ; du côté français, on trouve un comparable risque d'appropriation annexionniste dans le choix du libraire, tout comme à la précision apportée ("puisqu'elle a aussi écrit en français») est sous-jacente l'autre option si présente et dangereusement réductrice, celle de constamment renvoyer ces «inclassables » à leur statut étranger. Lise Gauvin a pointé cette polarisation et s'est interrogée sur la difficulté à «se situer entre ces deux extrêmes que sont l'intégration pure et simple au corpus français et la revalorisation exclusive de l'exotisme » (1997: 9 ; voir aussi Porra, 2011).

19 À l'égard du danger d'" exotisme ", il est clair que notre lecture de Nord Perdu à la lumière d'une poétique de la migrance implique le risque évident de cantonner l'œuvre de Nancy Huston à la thématique de la migrance et à celle du choix de la langue et donc à des éléments extérieurs au texte. Bien que ces thèmes soient évidemment présents, voire primordiaux notamment en ce qui concerne Nord Perdu, le péril existe d'ériger ce questionnement en une sorte de filtre biographique naiff et exclusif à travers lequel les écrits de Nancy Huston devraient être obligatoirement approchés.

Dans cette perspective, il me semble moins enfermant/plus pertinent d'aborder l'essai non pas (exclusivement) sous l'étiquette (sociologique) de "poétique de la migrance", mais d'axer autant sur le type d'écriture que l'analyse a permis de dégager et que j'ai qualifié plus haut de paradoxal ${ }^{4}$. Focaliser sur l'écriture paradoxale à l'œuvre dans Nord Perdu qui, dans une belle mise en abyme, se reflète dans le rapport paradoxal qui lie le moi (moderne) à la langue ("Oui, je n'ai qu'une langue, or ce n'est pas la mienne», Derrida, 1996 : 15, en italiques dans le texte), c'est inscrire le texte de manière décisive dans les esthétiques contemporaines. Ce faisant, c'est mettre au premier plan la littérarité de l'écriture hustonienne. De même, en ayant insisté sur la fragmentation, sur la contradiction et, par voie de conséquence, sur la présence d'un Moi pluriel toujours en quête, ces différents éléments en œuvre dans Nord Perdu rapprochent encore notre texte de l'écriture contemporaine dans le sens où celle-ci se caractérise aussi par le recentrement sur un je fragmenté, éparpillé mettant en scène une vision éclatée de la subjectivité et l'idée d'une identité toujours en devenir. C'est un peu comme si le vertigineux nombre de références présentes dans Nord Perdu nous rappelait cet héritage 
et/ou cette proximité avec le «Moi moderniste» (E. Saïd) : Samuel Beckett, Marica Bodroižić, T.S. Eliot, Annie Ernaux, Romain Gary, Vladimir Nabokov, Georges Perec, Sylvia Plath, Marcel Proust, Rainer Maria Rilke, Nathalie Sarraute, Marina Tsvetaïeva, Virginia Woolf, Marguerite Yourcenar, ... ces auteurs, tout comme Nancy Huston, se distinguent par un jeu, sinon une interrogation des limites entre réalité et fiction, entre vrai et faux. Si ces références intertextuelles listées relèvent de ce qui est parfois qualifié de «fiction intime », la désignation est entourée d'un flou théorique, rendu par une terminologie fluctuante - «œuvres postmodernes"; « essais-fictions"; «nouvelle fiction»; " autofictions » - et elle témoigne de ce fait d'une difficulté à classer ces écrits d'un point de vue générique... De même, si l'on considère Nord perdu, sous cet angle, est-ce un essai, un journal intime, un pamphlet, un récit autobiographique? C'est, ici encore, l'indétermination générique qui semble bien être le trait le plus caractéristique du texte. C'est donc, ici encore, évoquer une difficulté, voire une impossibilité à classer.

Embarras ou paradoxe quant à la classification, classification à la fois nécessaire à la pensée et impossible, c'est ce à quoi nous invite à réfléchir Perec par son texte Penser/ classer :

Comme si l'interrogation déclenchée par ce «PENSER/CLASSER » avait mis en question le pensable et le classable d'une façon que ma "pensée » ne pouvait réfléchir qu'en s'émiettant, se dispersant, qu'en revenant sans cesse à la fragmentation qu'elle prétendait vouloir mettre en ordre. Ce qui affleurait était tout entier du côté du flou, du flottement, du fugace, de l'inachevé (...) Que me demande-t-on au juste? Si je pense avant de classer ? Si je classe avant de penser? Comment je classe ce que je pense? Comment je pense quand je veux classer? (Perec (1982), $2003: 150 ; 151)$

Je suis consciente des pirouettes que je propose en passant d'une classification du texte ("poétique de la migrance») à une nouvelle ("écriture contemporaine »/écritures intimes). L'expression « écriture paradoxale » a, selon moi, l'avantage de ne pas centrer d'emblée sur une réalité biographique de l'auteur (migrance renvoyant bien au fait que celui-ci ait migré). Je parle ici (surtout) depuis mon expérience d'enseignante de littérature avec des étudiants des premières années. Ce que j'ai pu observer dans ce cadre, c'est que, pour ces (jeunes) lecteurs, la résistance est souvent immense à intégrer l'enseignement de Proust sur le leurre de l'approche étriquée du biographique. Dans le cas des auteurs francophones venus d'ailleurs s'ajoute à cette résistance un deuxième obstacle, tout aussi difficile à surmonter : la représentation romantique de la langue selon laquelle celle-ci est essentialisée, fétichisée en langue maternelle (Grutman, 2005 : 113) qui reste très présente chez le public que je rencontre. Penser le paradoxe derridien (« Oui, je n'ai qu'une langue, or ce n'est pas la mienne ") est un défi - or, les étudiants que je rencontre, dans leur grande majorité, se rapprochent de la stratégie du puriste face à l'inappropriable linguistique... Aussi il me semble judicieux de mettre d'abord la focale sur des questions d'écriture - esthétique. En d'autres termes, les phénomènes de la migration et du plurilinguisme constituent par moments des filtres de lecture réducteurs empêchant la pleine reconnaissance littéraire de Nord Perdu au sein de la littérature contemporaine. 


\section{BIBLIOGRAPHIE}

DELBART, Anne- Rosine (2005). Les exilés du langage. Un siècle d'écrivains français venus d'ailleurs

(1919-2000). Limoges : Pulim.

DERRIDA, Jacques (1996). Le monolinguisme de l'autre ou la prothèse d'origine. Paris : Galilée.

GAUVIN, Lise (1997). L'Écrivain francophone à la croisée des langues. Entretiens. Paris : Karthala.

GAUVIN, Lise (2005). « Surconscience linguistique », in Michel Beniamino \& Lise Gauvin (éds.),

Vocabulaire des études francophones. Les concepts de base. Limoges : Pulim, pp. 172-174.

GRUTMAN, Rainier (2005). « Langue maternelle/paternelle », in Michel Beniamino \& Lise Gauvin

(éds.), Vocabulaire des études francophones. Les concepts de base. Limoges : Pulim, pp. 113-114.

HUSTON, Nancy (1999). Nord perdu. Paris : Actes Sud.

JENNY, Laurent (2005). « La langue, le même et l'autre », Fabula-LHT, février 2005

<URL : http://www.fabula.org/lht/0/jenny.html>

KRISTEVA, Julia (1995). « Bulgarie, ma souffrance », L’infini, 51, pp. 42-52.

MATHIS-MOSER, Ursula \& MERTZ BAUMGARTNER, Birgit (2012). Passages et ancrages en France.

Dictionnaire des écrivains migrants de langue française (1981-2011). Paris : Honoré Champion.

PEREC, Georges (2003). Penser/classer. Paris : Seuil.

PORRA, Véronique (2011). Langue française, langue d'adoption. Une littérature 'invitée', entre création, stratégies et contraintes (1946-2000). Hildesheim/Zurich/New York : Georg Olms Verlag.

\section{NOTES}

1. Cette thématique centrale invite à évoquer la « surconscience linguistique ", concept forgé par Lise Gauvin (2005) et dont la présence renforce l'inscription de Nord Perdu dans une " poétique de la migrance ». L'essai hustonien y réfère en évoquant la « conscience exacerbée du langage » qu'a «l'étranger » et qui « peut être extrêmement propice à l'écriture » (Huston, 1999 : 43) ; plus loin dans le texte, il est question d' "extrême sensibilité linguistique», voire de "sensiblerie linguistique » (Huston, 1999 : 48), formulations que l'on doit également rapprocher du concept de la « surconscience linguistique ».

2. Voir également l'affirmation : «Proust n'est pas seulement un grand écrivain français, c'est le spécialiste inégalable des français » (Huston, 1999 : 43).

3. La polyphonie contradictoire est à l'œuvre dans l'ensemble du texte, elle est bien sa marque prépondérante. Qu'elle soit illustrée par le passage qui suit, tiré du chapitre "Orientation». L'étonnement naïf y est mêlé à des références implicites à la conscience moderne du mot, toujours insuffisant. Exposant une réflexion sur la difficulté à traduire Nord perdu en anglais, la narratrice commente: "Les dictionnaires nous induisent en confusion, nous jettent dans l'effroyable magma de l'entre-deux langues, là où les mots ne veulent pas dire, là où ils refusent de dire, là où ils commencent à dire une chose et finissent à dire une tout autre. Ce que l'on avait l'intention de dire. En principe. Ce que j'étais censée dire, si j'avais quelque chose à dire. Le Nord, j'en viens (...) (Huston, 1999 : 12-13). 
4. Faisant preuve d'une honnêteté intellectuelle qui force l'estime, l'éditrice du Dictionnaire des écrivains migrants relate le profond désaccord que sa démarche avait suscité auprès d'un auteur selon lequel la perspective sociologique défendue par le dictionnaire serait vaine, fausse et idéologiquement suspecte : «(...) c'est l'usage poétique de la langue qui fait les dictionnaires et non pas la sociologie. Le terme " littérature migrante » cloisonne les écrivains, les met dans des tiroirs, les discrimine car 'littérature migrante' n'est qu'un autre mot pour littératures francophones. Pourquoi l'origine, la vie, seraient -elles des critères d'inclusion ou d'exclusion? Pourquoi lui, en tant qu'écrivain, serait-il exclu de notre dictionnaire? En 'migrant' de la Bretagne à Paris il aurait vécu exactement le même dépaysement que mes auteurs soi-disant migrants...La littérature actuelle en langue française, ce sont les productions littéraires d'une génération d'écrivains qui n'ont que faire de la théorie, qui se révoltent contre les étiquettes et qui ont redécouvert la narration et la qualité poétique de la langue. Bref, en littérature, le regard sociologique est inadéquat per se et - disons-le - faux, inacceptable... " (Dictionnaire des écrivains migrants de langue française, 2011 : 53). La critique concerne le critère sociologique d'abord mais aussi, dans une perspective plus générale, est mise en question ici l'utilité / le sens même de la classification.

\section{RÉSUMÉS}

La focale de cet article est portée sur l'essai Nord perdu de Nancy Huston. Publié en 1998 dans le quotidien Parisien Le Monde, puis en volume l'année suivante, il s'agit d'un texte fréquemment cité par la critique. Il est lu ici sous le prisme d'une "poétique de la migrance ", en concentrant l'analyse sur trois de ses éléments clés, l'hybridité générique, l'hybridité textuelle et l'intertextualité. La thématique prépondérante, l'interrogation de la langue/des langues, assure au texte une polyphonie contradictoire parfaitement assumée : d'une part, Nord perdu donne voix aux différents types de rapports que l'on peut avoir face à l'expérience que la langue - et même si je la dis 'mienne' - irréductiblement échappe; d'autre part, l'essai ne cesse de mêler ces différentes attitudes qu'engendre l'« inappropriable linguistique » (Laurent Jenny). Il en résulte une écriture avant tout paradoxale, foncièrement moderne qui, en défiant toute tentative de classement, est un défi à la pensée.

This article examines the essay Losing north: musings on land, tongue and self by Nancy Huston. This text, published in 1998 in the Parisian daily newspaper Le Monde, then in a volume the following year, is frequently quoted by critics. It is read here through the lenses of migration poetry, focusing the analysis on three of its key elements: generic hybridity, textual hybridity and intertextuality. The major theme, the exploration of language/languages, endows the text with a contradictory polyphony which it embodies perfectly. On the one hand, Losing north: musings on land, tongue and self gives voice to the different types of relationships one may form faced with experiences which language, even if we claim it as our own, inevitably fails to capture satisfactorily. On the other hand, the essay persistently blends the different attitudes engendered by "inappropriable linguistics" (Laurent Jenny). The result is a type of writing which is above all else paradoxical, thoroughly modern and which, denying all forms of categorization, challenges thought. 
INDEX

Keywords : polyphony, poetry of migrance, textual and generic hybridity, intertextuality, paradoxical writing

Mots-clés : polyphonie, poétique de la migrance, hybridité textuelle et générique, intertextualité, écriture paradoxale

\section{AUTEUR}

\section{BRITTA BENERT}

Université de Strasbourg

britta.benert[at]unistra.fr 